\title{
Holling's "hungry mantid" model for the invertebrate functional response considered as a Markov process. III. Stable satiation distribution
}

\author{
H. J. A. M. Heijmans \\ Centre for Mathematics and Computer Science, Amsterdam \\ P.O. Box 4079, NL-1009 AB Amsterdam, The Netherlands
}

\begin{abstract}
In this paper, we study an analytical model describing predatory behaviour. It is assumed that the parameter describing the predator's behaviour is its satiation. Using semigroup methods and compactness arguments we prove that a stable satiation distribution is reached if $t \rightarrow \infty$. Furthermore, using a Trotter-Kato theorem we justify the transition to the much simpler problem that is obtained if the prey biomass tends to zero.
\end{abstract}

Key words: Satiation - functional response - forward equation - backward equation - positive operator - semigroup - trotter-Kato theorem

\section{Introduction}

In his famous paper [9], Holling described a detailed simulation model for the prey-catching behaviour of the praying mantid Hierodula crassa. One of his main purposes was to gain information about the qualitative and quantitative behaviour of the functional response of an invertebrate predator. (The functional response can be defined as the number (or total weight) of prey eaten per unit of time per predator as a function of the prey (prey biomass) density.)

In a series of papers $[13,14,15]$ Metz and van Batenburg presented an analytic reformulation of the theory of predation as propounded by Holling. They started by showing that Holling's assumptions implied that the predator's minimal state space is two-dimensional. More precisely: at every instant the state of the predator can be described by two parameters, its satiation (or gut content) $S$, and the maximum time $T$ still to be spent handling the prey. By the phrase "handling the prey" is meant pursuing it and (in case of a successful strike) eating it. As long as the predator is searching for his meal, $T=0$. A complete description of the predator's behaviour as a journey through this two-dimensional state space, can be found in [14].

Metz and van Batenburg also described several ways to simplify this rather complicated model. One possibility is to neglect handling time. The resulting "gobbler" model is just simple enough to be amenable to detailed analytical 
treatment and yet retains the essential stochastic features of the full model. The simplification can be justified if the handling time is relatively small in comparison with the searching time. In this paper we shall restrict ourselves to the gobbler model.

One of the main features of the gobbler model is that its state space is one-dimensional, the relevant parameter being the satiation $S$. Between two captures $S$ decreases continuously according to some ordinary differential equation $d S / d t=f(S)$ describing digestion, where we shall assume that $f(s)=$ $-a s$, as this seems a realistic assumption from a biological point of view. (See $[6,14])$.

Prey capture is a random event resulting in an instantaneous transition $S \rightarrow S+w$, where $w$ denotes prey weight (which is assumed to be constant). This jump causes a term with non-local argument in the balance equation for the $S$-distribution. (See Sect. 1.) The rate of prey capture depends (in a decreasing manner) on the satiation. (In the case of Holling's praying mantid this is due to the fact that its search field decreases with increasing satiation.)

This paper, which is self-contained, deals with a number of mathematical questions raised in the papers of Metz and van Batenburg [13, 14, 15]. These questions are formulated in the first section.

Our starting point is the so-called backward equation which is the adjoint of the balance equation for the probability density, or forward equation. This backward equation happens to be more tractable from a mathematical point of view, and it has a straightforward interpretation. In this manner we are able to prove that a stable satiation distribution is reached in the course of time.

Finally we refer to [4] where one uses techniques very similar to ours, to analyse a problem which is completely different from a biological point of view.

\section{The equations and their interpretation}

One of the equations proposed by Metz and van Batenburg $[13,14,15]$, as part of their model for predatory behaviour is:

$$
\frac{\partial p(s, t)}{\partial t}=-\frac{\partial}{\partial s}(f(s) p(s, t))-x g(s) p(s, t)+x g(s-w) p(s-w, t),
$$

where one should read $x g(s-w) p(s-w, t)=0$ if $s-w \leqslant 0$. Here $t$ denotes time, $s$ the predator's satiation, and $p(s, t)$ is the (unknown) probability density of $S$, i.e.

$$
\int_{s_{1}}^{s_{2}} p(s, t) d s=P\left\{s_{1}<S(t) \leqslant s_{2}\right\}
$$

is the probability that $S$ at time $t$ is between $s_{1}$ and $s_{2} . w$ is the weight (of the edible portion) of a prey, which is assumed to be constant for all prey. $f(s)$ is the digestion rate, which has been discussed in the Introduction and there it was assumed that $f(s)=-a s$. By a scaling of the time we may set $a=1 . x$ is the effective prey density and $x \cdot g(s)$ stands for the rate of prey capture, if the predator's satiation is $s$. It is assumed that there exists a value $c>0$ such that 
$g(s)=0$ if $s \geqslant c$. $c$ is called the satiation threshold. Accordingly we impose the boundary condition

$$
p(s, t)=0, \quad s \geqslant c+w .
$$

Furthermore we supplement (1.1a) with the initial condition

$$
p(s, 0)=p_{0}(s) .
$$

Because of the fact that (1.1a) describes the state of one single predator, we should have

$$
1=\int_{0}^{c+w} p_{0}(s) d s=\int_{0}^{c+w} p(s, t) d s, \quad t>0 .
$$

The second of these identities can be verified by integration of (1.1a) along the $s$-interval $[0, c+w]$.

In this paper we shall make the following assumption on $g$ :

(A) $g$ is a Lipschitz-continuous function on $[0, c+w], g$ is non-increasing and $g(s)=0$, if $s \geqslant c$.

Metz and van Batenburg [14] showed that for Holling's mantid model

$$
g(s)=\alpha\left(1-\frac{s}{c}\right)^{+} \cdot \exp \left(-b^{\prime}\left(1-\frac{s}{c^{\prime}}\right)^{+}\right)
$$

where $c^{\prime}<c$ and $\alpha, b^{\prime}$ are positive constants. The superscript ${ }^{+}$means that negative values are to be replaced by zero.

Remark 1.1: If (1.1 a) is formally integrated from $s$ to $c+w$, one obtains a partial differential equation for the distribution function of $S$ which necessarily is of bounded variation. This feature will be exploited in Sect. 5 .

In the literature $(1.1)$ is called the forward equation. (See e.g. [2,5].) The associated backward equation (or adjoint equation) is given by

$$
\frac{\partial n(s, t)}{\partial t}=-s \frac{\partial n(s, t)}{\partial s}-x g(s) n(s, t)+x g(s) n(s+w, t),
$$

where $x g(s) n(s+w, t)=0$ if $s>c$, and where we have substituted $f(s)=-s$. The backward equation is easier to derive in a rigorous manner directly from the constructive specification of the stochastic process and it is easier to handle as well. The main reason for this is that the backward equation has to be solved in the space of continuous functions, and the forward equation in the space of Borel measures. Below we shall briefly describe the duality relation between solutions of the forward and the backward equation.

Let $p\left(s, t ; p_{0}\right)$ be the solution of $(1.1)$, and let $n(s, t ; \phi)$ be the solution of (1.4), obeying the initial condition

$$
n(s, 0)=\phi(s),
$$

where $\phi$ is some continuous function on the interval $[0, c+w]$. (Here we have tacitly assumed that these solutions do exist. This is proved in Sect. 3.) Then

$$
\int_{0}^{c+w} p\left(s, t ; p_{0}\right) \phi(s) d s=\int_{0}^{c+w} p_{0}(s) n(s, t ; \phi) d s, \quad t \geqslant 0 .
$$


As a matter of fact this relation defines the solution of the forward equation, if the solution of the backward equation can be found for all continuous initial functions $\phi$.

Our starting point will be the backward equation. We shall prove existence and uniqueness of solutions of (1.4)-(1.5), and study the large-time behaviour of these solutions. Subsequently we shall interpret the results in terms of the forward equation.

Let $X=C[0, c+w]$ be the space of continuous functions on $[0, c+w]$ endowed with the usual sup-norm. We can rewrite (1.4)-(1.5) as an abstract Cauchy problem:

$$
\frac{d n}{d t}=A_{w} n, \quad n(0)=\phi \in X,
$$

where the closed operator $A_{w}$ on $X$ is defined by

$$
\left(A_{w} \psi\right)(s)=-s \frac{d \psi}{d s}-x g(s) \psi(s)+x g(s) \psi(s+w),
$$

for all $\psi$ in the domain of definition $\mathscr{D}\left(\boldsymbol{A}_{w}\right)$ of $\boldsymbol{A}_{w}$, which is given by

$$
\begin{array}{r}
\mathscr{D}\left(A_{w}\right)=\{\psi \in X \mid \psi \text { is absolutely continuous and the function } \\
\qquad s \rightarrow-s d \psi / d s(s) \text { defines an element of } X\} .
\end{array}
$$

Remark 1.2: The subscript $w$ accounts for the dependence of $\boldsymbol{A}_{w}$ on the prey weight $w$. As a matter of fact, the operator $\boldsymbol{A}_{w}$ also depends on the prey density $x$, but this is not expressed explicitly in our notation.

In Sect. 2 we shall investigate the spectrum of $A_{w}$, and in Sect. 3 we shall concentrate on the Cauchy problem (1.7).

In order to obtain more explicit results, Metz and van Batenburg [13, 14, 15] formally took the limit $w \rightarrow 0, x \rightarrow \infty, \xi=x w$ remaining constant. It appears that in the limit the mantid's catching behaviour becomes deterministic. Moreover, the limiting equation can be solved explicitly. One of the questions that one should answer is whether solutions of the original equation $(w>0)$ converge to solutions of the limiting equation $(w=0)$ if $w \rightarrow 0$. In Sect. 4 , we shall deal with this question. In Sect. 5 we shall give a rather detailed description of the relation between solutions of the forward and the backward equation.

An important biological quantity to be derived from the model is $W$, i.e. the total weight of prey caught per unit of time. The expectation $\mathscr{E} W$ of $W$ obeys the ordinary differential equation

$$
\frac{d \mathscr{E} W}{d t}=x w \int_{0}^{c+w} g(s) p(s, t) d s .
$$

Remark 1.3: In $[14,15]$ where one discusses the full stochastic model it is shown that

$$
\frac{d \mathscr{E} N}{d t}=x \int_{0}^{c+w} g(s) p(s, t) d s,
$$

where $N$ is the number of prey caught per unit of time. This is equivalent to (1.10) because $W=w N$. 
Metz and van Batenburg $[13,14,15]$ also derived an inhomogeneous partial differential equation from which the variance of $W$ can be obtained:

$$
\begin{aligned}
\frac{\partial z(s, t)}{\partial t}= & \frac{\partial}{\partial s}(s z(s, t))-x g(s) z(s, t)+x g(s-w) z(s-w, t) \\
& +x g(s-w) p(s-w, t)-x p(s, t) \int_{0}^{c+w} g(s) p(s, t) d s .
\end{aligned}
$$

This equation must be supplemented with boundary and initial conditions:

$$
\begin{aligned}
& z(s, t)=0, \quad s \geqslant c+w, \\
& z(s, 0)=0 .
\end{aligned}
$$

Remark 1.4: It was explained in $[13,15]$ how $\operatorname{var}(N)$ can be computed from $z(s, t)$. A straightforward computation using the results of $[13,15]$ shows that

$$
\begin{aligned}
\frac{d}{d t} \operatorname{var}(W) & =2 x w \cdot \operatorname{cov}[W, g(S)]+w \mathscr{E} g(S) \\
& =2 x w \cdot \operatorname{cov}[W, g(S)]+w \frac{d \mathscr{E} W}{d t}
\end{aligned}
$$

and

$$
\operatorname{cov}[W, g(S)]=w \int_{0}^{\infty} g(s) z(s, t) d s .
$$

In Sect. 6, we shall study (1.11).

If we let $t \rightarrow \infty$ in (1.10), we find an expression for the functional response $\Phi_{w}(\xi)$ (if we can prove convergence of the $S$-distribution towards a stationary state) which is the total biomass of prey caught per unit of time per predator in the stationary situation. Here $\xi=x w$, i.e. the density of prey biomass. It seems hard to obtain analytic results on the qualitative behaviour of $\Phi_{w}(\xi)$ in the most general case. However, it can be proved that for all $\xi>0, \lim _{w \downarrow 0} \Phi_{w}(\xi)=\Phi_{0}(\xi)$, where $\Phi_{0}$ can be obtained explicitly from the limiting equation studied in Sect. 4. Furthermore we are able to compute $\Phi_{w}(\xi)$ explicitly in the rather unrealistic special case that $c \leqslant w$. These results are given in Sect. 7 .

\section{The eigenvalue problem}

In this section we shall investigate the spectrum of the operator $\boldsymbol{A}_{w}$ defined by (1.8)-(1.9). It appears that the techniques which we shall use are in many regards similar to those in [8], where we studied the eigenvalue problem associated with a model for cell growth.

We use the following notation. For an operator $L$ we denote by $\sigma(L)$ and $P \sigma(L)$ the spectrum and point spectrum of $L$ respectively. $\rho(L)$ is the resolvent set, and $r(L)$ the spectral radius. $N(L)$ and $R(L)$ are the nullspace and range of $L$, and $\operatorname{ind}(L)=\operatorname{dim} N(L)-\operatorname{codim} R(L)$ is called the Fredholm index of $L$. (cf. $[12,18])$. 
Let $h \in X$. The inhomogeneous equation $\lambda \psi-A_{w} \psi=h$ can be rewritten as

$$
\lambda \psi(s)+s \frac{d \psi}{d s}+x g(s) \psi(s)-x g(s) \psi(s+w)=h(s) .
$$

Let

$$
E(s)=\exp \left(\int_{w}^{s} \frac{x g(\sigma)}{\sigma} d \sigma\right)
$$

It is obvious that

$$
E(s)=s^{\gamma x} \tilde{E}(s),
$$

where $\tilde{E}(s)$ is continuous on $[0, c+w]$, and satisfies $\tilde{E}(0)>0$. Here

$$
\gamma=g(0)
$$

Let

$$
\Omega=\{\lambda \in \mathbb{C} \mid \gamma x+\operatorname{Re} \lambda>0\} .
$$

Suppose that $\lambda \in \Omega$. Substitution of

$$
\phi(s)=s^{\lambda} E(s) \psi(s)
$$

in $(2.1)$ yields

$$
\frac{1}{s^{\lambda-1} E(s)} \frac{d \phi}{d s}-x g(s) \psi(s+w)=h(s)
$$

or equivalently,

$$
\frac{d \phi}{d s}-x g(s) s^{\lambda-1} E(s) \psi(s+w)=h(s) s^{\lambda-1} E(s) .
$$

It follows from (2.6) and (2.3) that $\phi(s)=s^{\lambda} E(s) \psi(s)=s^{\lambda+\gamma x} \tilde{E}(s) \psi(s)$. Now $\lambda \in \Omega$ and the continuity of $\psi$ imply that $\phi(0)=0$. Integrating (2.7) from 0 to $s$ and plugging (2.6) back into the result yields:

$$
\psi(s)-\frac{x}{s^{\lambda} E(s)} \int_{0}^{s} g(\sigma) \sigma^{\lambda-1} E(\sigma) \psi(\sigma+w) d \sigma=\frac{1}{s^{\lambda} E(s)} \int_{0}^{s} h(\sigma) \sigma^{\lambda-1} E(\sigma) d \sigma .
$$

Let the linear operators $\hat{T}_{\lambda}$ and $U_{\lambda}$ on $X$ be defined by

$$
\begin{aligned}
& \left(\hat{T}_{\lambda} \rho\right)(s)=\frac{x}{s^{\lambda} E(s)} \int_{0}^{s} g(\sigma) \sigma^{\lambda-1} E(\sigma) \rho(\sigma+w) d \sigma \\
& \left(U_{\lambda} \rho\right)(s)=\frac{1}{s^{\lambda} E(s)} \int_{0}^{s} \sigma^{\lambda-1} E(\sigma) \rho(\sigma) d \sigma
\end{aligned}
$$

for all $\rho \in X$.

It is obvious that $\hat{T}_{\lambda}$ and $U_{\lambda}$ are bounded. Now (2.8) can be rewritten as

$$
\psi-\hat{T}_{\lambda} \psi=U_{\lambda} h
$$

The following result is straightforward. 
Lemma 2.1. Let $\lambda \in \Omega$ and $h \in X$. Then $\psi \in X$ is a solution of the inhomogeneous equation $\lambda \psi-A_{w} \psi=h$ if and only if $\psi-\hat{T}_{\lambda} \psi=U_{\lambda} h$.

Thus the inhomogeneous equation (2.1) involving the unbounded operator $A_{w}$ can be reformulated in terms of the bounded operators $\hat{T}_{\lambda}$ and $U_{\lambda}$. A closer look on (2.8) makes clear that it suffices to study this equation on the subinterval $[w, c+w]$, because knowledge of $\psi$ on this subinterval would enable us to compute $\left(\hat{T}_{\lambda} \psi\right)(s)$ for all $s \in[0, c+w]$. Let

$$
X_{1}=C[w, c+w]
$$

with the supnorm. For $\psi \in X_{1}$ we define $T_{\lambda} \psi$ in the following way. Let $\hat{\psi} \in X$ such that $\hat{\psi}(s)=\psi(s), s \in[w, c+w]$, then $\left(T_{\lambda} \psi\right)(s):=\left(\hat{T}_{\lambda} \hat{\psi}\right)(s), s \in[w, c+w]$. Observe that $T_{\lambda}: X_{1} \rightarrow X_{1}$ is well-defined, i.e. $T_{\lambda} \psi$ does not depend on the choice of $\hat{\psi}$. [18]).

The following result can be established using the Arzelà-Ascoli theorem (cf.

Lemma 2.2. $T_{\lambda}: X_{1} \rightarrow X_{1}$ is compact.

Let

$$
\Sigma=\left\{\lambda \in \Omega \mid 1 \in \operatorname{P\sigma }\left(T_{\lambda}\right)\right\} .
$$

Theorem 2.3. $\sigma\left(A_{w}\right) \cap \Omega=P \sigma\left(A_{w}\right) \cap \Omega=\Sigma$.

Proof: Let $\lambda \in \Omega$. The homogeneous equation $A_{w} \psi=\lambda \psi$ can be rewritten as $\hat{T}_{\lambda} \psi=\psi$. Let $\tilde{\psi}$ be the restriction of $\psi$ to $[w, c+w]$, then $T_{\lambda} \tilde{\psi}=\tilde{\psi} . \tilde{\psi}=0$ would imply $\hat{T}_{\lambda} \psi=\psi=0$. As a consequence, if $\lambda \in P \sigma\left(A_{w}\right)$, then $\lambda \in \Sigma$. Similar arguments yield that $\lambda \in \Sigma$ implies that $\lambda \in P \sigma\left(A_{w}\right)$. Now suppose that $\lambda \in \Omega \cap \sigma\left(A_{w}\right)$. The inhomogeneous equation $\lambda \psi-A_{w} \psi=h$, where $h \in X$, is equivalent to $\psi-\hat{T}_{\lambda} \psi=$ $U_{\lambda} h$. Suppose that $\lambda \notin \operatorname{P\sigma }\left(A_{w}\right)$, then we have $1 \notin \operatorname{P\sigma }\left(T_{\lambda}\right)$ yielding that the equation $\psi-\hat{T}_{\lambda} \psi=U_{\lambda} h$ can be solved on the interval $[w, c+w]$. Its solution is $\psi(s)=$ $\left(\left(I-T_{\lambda}\right)^{-1} U_{\lambda} h\right)(s), s \in[w, c+w]$. For $s \in[0, w]$ we find $\psi(s)=\left(\hat{T}_{\lambda} \psi\right)(s)+\left(U_{\lambda} h\right)(s)$ where we have exploited the fact that $\left(\hat{T}_{\lambda} \psi\right)(s)$ can be computed on $[0, w]$ if $\psi(s)$ is known on $[w, c+w]$. This proves the result.

We shall need the following lemma in the proof of Theorem 2.11.

Lemma 2.4. $R\left(\lambda I-A_{w}\right)$ is closed if $\lambda \in \Omega$.

Proof: Suppose $h_{n} \in R\left(\lambda I-A_{w}\right)$ and $h_{n} \rightarrow h, n \rightarrow \infty$. Let $\psi_{n}$ be such that $\lambda \psi_{n}-$ $A_{w} \psi_{n}=h_{n}$. Lemma 2.1 yields that $\psi_{n}-\hat{T}_{\lambda} \psi_{n}=h_{n}$. Let $\tilde{\psi}_{n}$ and $\tilde{h}_{n}$ be the restriction of $\psi_{n}$ respectively $h_{n}$ to $[w, c+w]$. Thus $\tilde{\psi}_{n}-T_{\lambda} \tilde{\psi}_{n}=\tilde{h}_{n}$. Hence $\tilde{h}_{n} \in R\left(I-T_{\lambda}\right)$ and $\tilde{h}_{n} \rightarrow \tilde{h}, n \rightarrow \infty$ where $\tilde{h}$ denotes the restriction of $h$ to $[w, c+w]$. From the compactness of $T_{\lambda}$ we conclude that $R\left(I-T_{\lambda}\right)$ is closed. Therefore $\tilde{h} \in R\left(I-T_{\lambda}\right)$. Let $\tilde{\psi} \in X_{1}$ be such that $\tilde{\psi}-T_{\lambda} \tilde{\psi}=\tilde{h}$. We define $\psi$ by:

$$
\begin{gathered}
\psi(s)=\tilde{\psi}(s), s \in[w, c+w], \\
\psi(s)=\frac{x}{s^{\lambda} E(s)} \int_{0}^{s} g(\sigma) \sigma^{\lambda-1} E(\sigma) \tilde{\psi}(\sigma+w) d \sigma+\left(U_{\lambda} h\right)(s), s \in[0, w] .
\end{gathered}
$$

It is clear that $\psi$ is a solution of $\psi-\hat{T}_{\lambda} \psi=h$, hence $\lambda \psi-A_{w} \psi=h$. 
The following result is stated for the sake of completeness. We do not need it in our calculations.

Theorem 2.5. $\mathbb{C} / \Omega \subset \sigma\left(A_{w}\right)$.

Proof: Let $\lambda$ be such that $\gamma x+\operatorname{Re} \lambda<0$. Without loss of generality we may assume that $\lambda \in \mathbb{R}$. Let $p=-\gamma x-\lambda>0$. The homogeneous equation $A_{w} \psi=\lambda \psi$ can be solved on $[w, c+w]$ within a finite number of steps. Let $\bar{\psi}(s)$ be the solution on $[w, c+w]$. For $s \in[0, w]$ we must solve

$$
\frac{d \psi}{d s}+\frac{x g(s)+\lambda}{s} \psi(s)=\frac{x g(s) \bar{\psi}(s+w)}{s}, \quad \psi(w)=\bar{\psi}(w) .
$$

We obtain

$$
\begin{aligned}
\psi(s) & =\bar{\psi}(w) \cdot \frac{1}{s^{\lambda} E(s)}-\frac{x}{s^{\lambda} E(s)} \int_{s}^{w} g(\sigma) \sigma^{\lambda-1} E(\sigma) \bar{\psi}(\sigma+w) d \sigma \\
& =\frac{1}{\tilde{E}(s)}\left[s^{p} \bar{\psi}(w)-s^{p} \int_{s}^{w} g(\sigma) \sigma^{-p-1} \tilde{E}(\sigma) \bar{\psi}(\sigma+w) d \sigma\right]
\end{aligned}
$$

and it can be easily checked that this expression defines a continuous function if $p>0$. Therefore $\lambda \in \operatorname{P\sigma }\left(A_{w}\right)$ if $\operatorname{Re} \lambda+\gamma x<0$. This, and the closedness of the spectrum, yields the result.

The asymptotic behaviour of solutions of (1.4) for $t \rightarrow \infty$ appears to be determined by the dominant eigenvalue of $A_{w}$, i.e. the eigenvalue with the largest real part. As we did in [8], we use positive operator theory to characterize this dominant eigenvalue. We refer to the famous paper of Krein and Rutman [11], and the monograph of Schaefer [17]. See also [10]. Let

$$
\begin{gathered}
X_{1}^{+}=\left\{\psi \in X_{1} \mid \psi(s) \geqslant 0, w \leqslant s \leqslant c+w\right\}, \\
\Omega_{R}=\Omega \cap \mathbb{R} .
\end{gathered}
$$

Then $X_{1}^{+}$defines a closed, convex cone in $X_{1}$, and for all $\lambda \in \Omega_{R}$ we have that $T_{\lambda}$ is positive with respect to $X_{1}^{+}$, i.e.

$$
T_{\lambda} \psi \in X_{1}^{+} \quad \text { if } \psi \in X_{1}^{+} .
$$

In the sequel we need a stronger notion of positivity.

Definition [11]. An operator is called strongly positive if each nonzero element within the cone is mapped into the interior of that cone by some power of the operator.

Theorem 2.6. For all $\lambda \in \Omega_{R}, T_{\lambda}$ is strongly positive with respect to $X_{1}^{+}$.

Proof: Let $\lambda \in \Omega_{R}$ and $\psi \in X_{1}^{+}, \psi \neq 0$. There exists an $\bar{s} \in(w, c+w)$ and an $\varepsilon>0$ such that $\psi(s)>0, s \in(\bar{s}-\varepsilon, \bar{s}+\varepsilon)$. Now suppose that $s \geqslant \bar{s}-w$ and $s \in[w, c+w]$, then we have

$$
\left(T_{\lambda} \psi\right)(s) \geqslant \frac{x}{s^{\lambda} E(s)} \int_{s-w-\varepsilon}^{s} g(\sigma) \sigma^{\lambda-1} E(\sigma) \psi(\sigma+w) d \sigma>0 .
$$


Applying $T_{\lambda}$ once more yields

$$
\left(T_{\lambda}^{2} \psi\right)(s)>0, \quad s \geqslant \bar{s}-2 w .
$$

Hence if $p$ is the smallest integer satisfying $p \geqslant(c+w) / w$, then we have $\left(T_{\lambda}^{p} \psi\right)(s)>$ $0, s \in[w, c+w]$, yielding that $T_{\lambda}^{p} \psi \in \dot{X}_{1}$.

Now we can apply Theorem 6.3 of [11], and we obtain the following result. Let $X_{1}^{*}$ be the adjoint space of $X_{1}$, and let $\left(X_{1}^{+}\right)^{*}$ be the adjoint cone of $X_{1}^{+}$. (See e.g. [11].) With $T_{\lambda}^{*}$ we denote the adjoint operator of $T_{\lambda}$.

Lemma 2.7. For all $\lambda \in \Omega_{R}, r_{\lambda}=r\left(T_{\lambda}\right)$ is an algebraically simple eigenvalue of both $T_{\lambda}$ and $T_{\lambda}^{*}$. Furthermore there exist $a \psi_{\lambda} \in \dot{X}_{1}^{+}$and $F_{\lambda} \in\left(X_{1}^{+}\right)^{*}$ such that

$$
\begin{aligned}
T_{\lambda} \psi_{\lambda} & =r_{\lambda} \psi_{\lambda} \\
T_{\lambda}^{*} F_{\lambda} & =r_{\lambda} F_{\lambda}
\end{aligned}
$$

and $\psi_{\lambda}$ is the only positive eigenvector of $T_{\lambda}$. Moreover, $F_{\lambda}$ is strictly positive, i.e. $F_{\lambda}(\psi)>0$ for all $\psi \in X_{1}^{+} \backslash\{0\}$.

Now $\psi_{\lambda}$ is an eigenvector of $\boldsymbol{A}_{w}$ if and only if $r_{\lambda}=1$. We shall prove that $\lambda \in \Omega_{R}$ is uniquely determined by this condition. Obviously

$$
T_{0} 1=1 \text {, }
$$

where the function $l \in X_{1}$ is defined by $l(s)=1, s \in[w, c+w]$. Clearly $l \in X_{1}^{+}$ and we conclude from Lemma 2.7 that $r\left(T_{0}\right)=1$.

Lemma 2.8. $r\left(T_{\lambda}\right)$ is strictly decreasing in $\lambda \in \Omega_{R}$.

Proof: Suppose $\lambda, \mu \in \Omega_{R}$ and $\lambda>\mu$. A straightforward computation shows that

$$
\left(T_{\mu}-T_{\lambda}\right) \dot{X}_{1}^{+} \subseteq \dot{X}_{1}^{+} .
$$

In particular $\left(T_{\mu}-T_{\lambda}\right) \psi_{\lambda} \in \dot{X}_{1}^{+}$. From the strict positivity of $F_{\mu}$ we conclude that $\left\langle F_{\mu},\left(T_{\mu}-T_{\lambda}\right) \psi_{\lambda}\right\rangle>0$, or equivalently

$$
r_{\mu}\left\langle F_{\mu}, \psi_{\lambda}\right\rangle>r_{\lambda}\left\langle F_{\mu}, \psi_{\lambda}\right\rangle
$$

Therefore $r_{\mu}>r_{\lambda}$, and this proves the lemma.

Now we shall interpret the results in terms of $\boldsymbol{A}_{\boldsymbol{w}}$.

Theorem 2.9. $\lambda=0$ is an algebraically simple eigenvalue of $A_{w}$ with positive eigenvector 1. $A_{w}$ has no other positive eigenvectors. The eigenvalue $\lambda=0$ is strictly dominant, i.e. $\lambda \in \sigma\left(A_{w}\right), \lambda \neq 0 \Rightarrow \operatorname{Re} \lambda<0$.

Proof: From the geometric simplicity of the eigenvalue 1 of $T_{0}$ we conclude that $\lambda=0$ is a geometric simple eigenvalue of $A_{w}$. Now suppose that $A_{w} \psi=1$ for some $\psi \in X$. Then Lemma 2.1 yields that $\hat{T}_{0} \psi-\psi=U_{0} 1$. Hence $T_{0} \tilde{\psi}-\tilde{\psi}=\phi$ where $\tilde{\psi}$ and $\phi$ are the restrictions of $\psi$ respectively $U_{0} l$ to the interval $[w, c+w]$. We observe that $\phi \in \dot{X}_{1}^{+}$. The Fredholm alternative states that $F_{0}(\phi)=0$, where $F_{0}$ is given by (2.16b) for $\lambda=0$. However $F_{0}(\phi)>0$, which is a contradiction. Therefore 0 is an algebraically simple eigenvalue of $A_{w}$. The proof of strict dominance of the eigenvalue $\lambda=0$ is similar to the proof of Theorem 6.2 in [8]. 
The following result, stated in [12], enables us to give a more complete description of $\sigma\left(A_{w}\right) \cap \Omega$.

Lemma 2.10 [12]. Suppose $L$ is a closed linear operator on a Banach space $E$ having a dense domain. For all $\lambda \in \mathbb{C}$ satisfying the following conditions

(i) $\lambda$ is on the boundary of $\sigma(L)$,

(ii) $R(\lambda I-L)$ is closed,

(iii) $N(\lambda I-L)$ has a finite dimension,

we have ind $(\lambda I-L)=0$ and $\lambda$ is a pole of the resolvent.

Now we can prove:

Theorem 2.11. $\sigma\left(A_{w}\right) \cap \Omega$ consists entirely of eigenvalues $\lambda$ satisfying

(i) $\lambda$ is a pole of the resolvent,

(ii) $\operatorname{ind}\left(\lambda I-A_{w}\right)=0$.

Proof: Suppose that $\dot{\lambda} \in \sigma\left(A_{w}\right) \cap \Omega$ is on the boundary of $\sigma\left(A_{w}\right)$. Lemma 2.4 states that $R\left(\lambda I-A_{w}\right)$ is closed. From $\operatorname{dim} N\left(\lambda I-A_{w}\right)=\operatorname{dim}\left(I-T_{\lambda}\right)$ and the compactness of $T_{\lambda}$ we conclude that $\operatorname{dim} N\left(\lambda I-A_{w}\right)<\infty$. Now Lemma 2.10 states that $\lambda$ is an isolated eigenvalue of $A_{w}$. Hence every boundary point of $\sigma\left(A_{w}\right) \cap \Omega$ is isolated. As a consequence there are two possibilities:

1) $\sigma\left(A_{w}\right) \cap \Omega=\Omega$,

2) $\sigma\left(A_{w}\right) \cap \Omega$ contains only isolated eigenvalues.

However, the existence of the dominant eigenvalue $\lambda=0$ excludes the first possibility. This proves the result.

Remark 2.1: We can also state our results in terms of normal eigenvalues and essential spectrum (in the sense of Browder) (See e.g. $[4,19]$.) Let $L$ be a closed linear operator on a Banach space. $\lambda \in \sigma(L)$ is called a normal eigenvalue of $L$ if

(a) $\lambda$ is an isolated element of $\sigma(L)$,

(b) $\operatorname{Ran}(\lambda I-L)$ is closed,

(c) The generalized eigenspace corresponding to $\lambda$ is finite-dimensional, i.e.

$$
\operatorname{dim}\left(\bigcup_{k=1}^{\infty} N(\lambda I-L)^{k}\right)<\infty
$$

It can be proved that every normal eigenvalue is an isolated pole of the resolvent of finite order. We denote the set of normal eigenvalues with $\sigma_{n}(L)$. The essential spectrum $\sigma_{e}(L)$ of $L$ is defined by $\sigma_{e}(L)=\sigma(L) \backslash \sigma_{n}(L)$. Now, our results can be reformulated as

$$
\sigma(A) \cap \Omega=\sigma_{n}(A), \quad \mathbb{C} \backslash \Omega=\sigma_{e}(A) .
$$

Our next step is the derivation of the so called characteristic equation which provides us with a tool to compute all eigenvalues of $A$ which are elements of $\Omega$. We shall not go into detail. The interested reader is referred to [8].

For all $\lambda \in \Omega$, the operator $T_{\lambda}$ can be decomposed in the following way.

$$
\begin{aligned}
\left(T_{\lambda} \psi\right)(s)= & \frac{x}{s^{\lambda} E(s)} \int_{0}^{c} g(\sigma) \sigma^{\lambda-1} E(\sigma) \psi(\sigma+w) d \sigma \\
& -\frac{x}{s^{\lambda} E(s)} \int_{s}^{c} g(\sigma) \sigma^{\lambda-1} E(\sigma) \psi(\sigma+w) d \sigma,
\end{aligned}
$$


which we rewrite as

$$
T_{\lambda} \psi=\left\langle H_{\lambda}, \psi\right\rangle e_{1}^{\lambda}+N_{\lambda} \psi
$$

where

$$
\begin{gathered}
e_{1}^{\lambda}(s)=\frac{1}{s^{\lambda} E(s)}, \quad s \in[w, c+w], \\
\left\langle H_{\lambda}, \psi\right\rangle=x \int_{0}^{c} g(\sigma) \sigma^{\lambda-1} E(\sigma) \psi(\sigma+w) d \sigma
\end{gathered}
$$

defines a bounded linear functional on $X_{1}$, and

$$
\left(N_{\lambda} \psi\right)(s)=\frac{-x}{s^{\lambda} E(s)} \int_{s}^{c} g(\sigma) \sigma^{\lambda-1} E(\sigma) \psi(\sigma+w) d \sigma
$$

defines a compact operator on $X_{1}$. Moreover $N_{\lambda}$ is nilpotent, i.e.

$$
N_{\lambda}^{p}=0 \text {, }
$$

where $p$ is the smallest integer such that $p \geqslant(c+w) / w$. Let

$$
e_{k}^{\lambda}=N_{\lambda}^{k-1} e_{1}^{\lambda}, \quad k=1, \ldots, p,
$$

then $e_{1}^{\lambda}, \ldots, e_{p}^{\lambda}$ are linearly independent vectors in $X_{1}$. By iteration of (2.18) we obtain

$$
T_{\lambda}^{p} \psi=\left\langle H_{\lambda}, T_{\lambda}^{p-1} \psi\right\rangle \cdot e_{1}^{\lambda}+\left\langle H_{\lambda}, T_{\lambda}^{p-2} \psi\right\rangle \cdot e_{2}^{\lambda}+\cdots+\left\langle H_{\lambda}, \psi\right\rangle \cdot e_{p}^{\lambda},
$$

implying that all eigenvectors of $T_{\lambda}$ can be written as a linear combination of $e_{1}^{\lambda}, \ldots, e_{p}^{\lambda}$. Now suppose that $T_{\lambda} \psi=\psi$ for some $\lambda \in \Omega$ and $\psi \in X_{1}$, then $\psi=$ $\psi_{1} e_{1}^{\lambda}+\cdots+\psi_{p} e_{p}^{\lambda}$ for some $\psi_{i} \in \mathbb{C}, i=1, \ldots, p$. Substitution of this expression in (2.18) and using (2.23) leads to the following identity:

$$
\left\langle H_{\lambda}, e_{1}^{\lambda}+\cdots+e_{p}^{\lambda}\right\rangle=1,
$$

which is called the characteristic equation.

Theorem 2.12. $\lambda \in \sigma\left(A_{w}\right) \cap \Omega$ if and only if $\left\langle H_{\lambda}, e_{1}^{\lambda}+\cdots+e_{p}^{\lambda}\right\rangle=1$. Every closed vertical strip inside $\Omega,\left\{\lambda \mid \xi_{1} \leqslant \operatorname{Re} \lambda \leqslant \xi_{2}\right\}$ where $\xi_{1} \leqslant \xi_{2}$, contains at most finitely many elements of $\sigma\left(A_{w}\right)$.

A similar result is proved in [8].

From Theorem 2.12 we conclude that there exists an $\varepsilon>0$ that

$$
\sigma\left(A_{w}\right) \cap\{\lambda \mid \operatorname{Re} \lambda \geqslant-\varepsilon\}=\{0\} .
$$

We end this section with a brief study of the adjoint operator of $A_{w}$. In the Appendix we shall prove that the adjoint operator $A_{w}^{*}$ defined on

$$
X^{*}=\{\Psi \mid \Psi \text { is a bounded variation function on }[0, c+w] \text { and } \Psi(c+w)=0\} \text {, }
$$

is given by

$$
\left(A_{w}^{*} \Psi\right)(s)=s \frac{d \Psi}{d s}(s)-x \int_{s-w}^{s} g(\sigma) d \Psi(\sigma),
$$


having a domain

$$
\mathscr{D}\left(A_{w}^{*}\right)=\left\{\Psi \in X^{*} \mid \Psi \text { is absolutely continuous and } s \rightarrow s \frac{d \Psi(s)}{d s} \in X^{*}\right\} .
$$

For $\Psi \in X^{*}$ and $\phi \in X$ we define

$$
\langle\Psi, \phi\rangle=\int_{0}^{c+w} \phi(s) d \Psi(s) .
$$

The following result is straightforward.

Theorem 2.13. If $\Psi$ is an eigenvector of $A_{w}^{*}$ corresponding to an eigenvalue $\lambda \in \Omega$, then $\Psi$ satisfies

$$
\Psi(s)=-s^{\lambda} \int_{s}^{c+w} \sigma^{-\lambda-1}\left(\int_{\sigma-w}^{\sigma} x g(\eta) d \Psi(\eta)\right) d \sigma .
$$

If $\lambda \neq 0$ then $\Psi(0)=0$. If $\lambda=0$ then $\Psi(0)<0$ and $\Psi$ is increasing.

Remark 2.2: Notice that for $\Psi \in X^{*}$ we have $\langle\Psi, 1\rangle=-\Psi(0)$.

Because of the algebraic simplicity of the dominant eigenvalue $\lambda=0$, and Theorem 2.11 we have the following invariant decomposition of $X$.

$$
X=N\left(A_{w}\right) \oplus R\left(A_{w}\right),
$$

and $N\left(A_{w}\right)=\{\alpha \cdot 1 \mid \alpha \in \mathbb{C}\}$.

Let $P$ be the projection on $N\left(A_{w}\right)$ corresponding with this decomposition, and let $\Psi_{w}$ be the eigenvector of $A_{w}^{*}$ associated with the dominant eigenvalue $\lambda=0$, and normalized by the condition $\Psi_{w}(0)=-1$, then

$$
P \psi=\left\langle\Psi_{w}, \psi\right\rangle \cdot 1
$$

Observe that $P 1=\left\langle\Psi_{w}, 1\right\rangle \cdot 1=-\Psi_{w}(0) \cdot 1=1$.

\section{The backward equation}

Here we shall examine the initial value problem (1.4)-(1.5), or equivalently (1.7). We obtain existence and uniqueness results by proving that $A_{w}$ generates a strongly continuous semigroup on $X$. The method of proof is very similar to the one used by Diekmann et al. in [4], where they investigate the evolution of a size-structured cell population reproducing by fission. (In [4] however, the forward equation is studied.) The idea is to integrate the partial differential equation along its characteristics and to use a variation-of-constants formula, and this will give us the solution as a series.

In the second part of this section, we prove a sort of asymptotic compactness result for the semigroup, which enables us to characterize the behaviour of the solutions for large $t$. 
$A_{w}$ as defined by (1.8)-(1.9) can be written as the sum of a closed and a bounded operator.

$$
A_{w}=B+C
$$

where

$$
\begin{aligned}
& (B \psi)(s)=-s \frac{d \psi}{d s}-x g(s) \psi(s), \\
& (C \psi)(s)=x g(s) \psi(s+w),
\end{aligned}
$$

where the domain $D(B)$ of $B$ is given by

$$
\mathscr{D}(B)=\left\{\psi \in X \mid \psi \text { is absolutely continuous and } s \rightarrow s \frac{d \psi}{d s} \text { is continuous }\right\} .
$$

A straightforward computation shows that $B$ generates a strongly continuous semigroup $S_{0}(t)$ given by

$$
\left(S_{0}(t) \psi\right)(s)=\frac{E\left(s e^{-t}\right)}{E(s)} \psi\left(s e^{-t}\right), \quad s \in[0, c+w], \quad t \geqslant 0 .
$$

Now a standard perturbation lemma (see e.g. [16]) yields that $A_{w}=B+C$ generates a strongly continuous semigroup as well.

Theorem 3.1. $A_{w}$ generates a strongly continuous semigroup $T_{w}(t)$.

One can prove this in the following way.

Consider $C n$ as the inhomogeneous part of the equation $d n / d t=B n+C n$, and apply the variation-of-constants formula. It follows that $n(t)$ has to be a solution of the integral equation

$$
n(t)=S_{0}(t) \phi+\int_{0}^{t} S_{0}(t-\tau) C n(\tau) d \tau
$$

The result follows from a standard contraction and continuation argument.

Remark 3.1: In [16] one uses the Hille-Yosida conditions to prove the result.

Now iteration gives us the solution $n(t)=T_{w}(t) \phi$ as a series

$$
T_{w}(t) \phi=\sum_{n=0}^{\infty} S_{n}(t) \phi, \quad t \geqslant 0,
$$

where this series converges in the operator norm. $S_{n}(t)$ is determined by the recurrent relation

$$
S_{n+1}(t) \phi=\int_{0}^{t} S_{0}(t-\tau) C S_{n}(\tau) \phi d \tau, \quad n=0,1,2, \ldots
$$

For the initial value problem (1.4)-(1.5) this means that there does exist a unique solution in the following sense. Let the operator $D$ on $C\left(\mathbb{R}^{+} x[0, c+w] \rightarrow \mathbb{R}\right)$ be given by

$$
(D n)(s, t)=\lim _{h \rightarrow 0} \frac{1}{h}\left(n\left(s e^{h}, t+h\right)-n(s, t)\right),
$$


then the initial value problem

$$
(D n)(s, t)=-x g(s) n(s, t)+x g(s) n(s+w, t), \quad n(s, 0)=\phi(s)
$$

has a unique solution. In other words the solution $n(s, t)=\left(T_{w}(t) \phi\right)(s)$ is differentiable along the characteristics of the partial differential equation (1.4). If moreover $\phi \in \mathscr{D}(A)$, then the solution is differentiable in $s$ and $t$ separately.

We are especially interested in the behaviour of the solutions $n(s, t)$ for large $t$. The characterization of this behaviour would be relatively easy if $T_{w}(t)$ were compact after finite time. (See [4].) Unfortunately $S_{0}(t)$ which contributes to $T_{w}(t)$ for all $t \geqslant 0$, never becomes compact. However, we can prove that this contribution becomes smaller and smaller.

Lemma 3.2. $\left\|S_{0}(t)\right\| \leqslant C e^{-\gamma x t}, t \geqslant 0$, for some positive constant $C$ not depending on $t$.

Proof: Let $\phi \in X,\|\phi\| \leqslant 1$.

$$
\begin{aligned}
\left|\left(S_{0}(t) \phi\right)(s)\right| & =\left|\frac{E\left(s e^{-t}\right)}{E(s)} \phi\left(s e^{-t}\right)\right| \\
& =\left|\frac{s^{\gamma x} e^{-\gamma x t} \tilde{E}\left(s e^{-t}\right)}{s^{\gamma x} \tilde{E}(s)} \phi\left(s e^{-t}\right)\right| \leqslant C e^{-\gamma x t}
\end{aligned}
$$

where we have used (2.3) and the fact that $\tilde{E}(s)$ is bounded from above and below.

Lemma 3.3. $U(t):=\sum_{n=1}^{\infty} S_{n}(t)$ is compact for all $t \geqslant 0$.

Proof: A simple calculation shows that

$$
\left(S_{1}(t) \phi\right)(s)=x \int_{0}^{t} g\left(s e^{-t+\tau}\right) \frac{E\left(s e^{-t+\tau}\right)}{E(s)} \frac{E\left(s e^{-t}+w e^{-\tau}\right)}{E\left(s e^{-t+\tau}+w\right)} \phi\left(s e^{-t}+w e^{-\tau}\right) d \tau .
$$

One can apply the Arzelà-Ascoli theorem (cf. [18]) to establish the compactness of $S_{1}(t)$, provided that the derivative of $g$ is bounded. Because of assumption $(A)$ this is indeed the case. Using recurrence relation (3.7), it follows immediately that $S_{n}(t)$ is compact for all $n \geqslant 1$. This and the convergence of the series (3.6) with respect to the norm topology yields the result.

Now let

$$
\nu:=\min \{\varepsilon, \gamma x\},
$$

where $\varepsilon$ is characterized by (2.26). Let $P$ be the projection on $N\left(A_{w}\right)$, given by (2.32)

Theorem 3.4. For all $\eta>0$ there exists a constant $K(\eta)>0$ such that

$$
\left\|T_{w}(t) \phi-P \phi\right\| \leqslant K(\eta) e^{-(\nu-\eta) t}\|\phi\|
$$

for all $\phi \in X$ and $t \geqslant 0$.

Proof: Let $\lambda \in \mathbb{C}$ be such that $\operatorname{Re} \lambda>-\gamma x$, hence $\left|e^{\lambda t}\right|>e^{-\gamma x t}$. Obviously $T_{w}(t)-$ $e^{\lambda t} I=S_{0}(t)+U(t)-e^{\lambda t} I$, where $U(t)=\sum_{n=1}^{\infty} S_{n}(t)$. Lemma 3.2 yields that $r\left(S_{0}(t)\right) \leqslant e^{-\gamma x t}$. Therefore $S_{0}(t)-e^{\lambda t} I$ is invertible. Thus $T_{w}(t)-e^{\lambda t} I=$ $\left(S_{0}(t)-e^{\lambda t} I\right)\left(I+\left(S_{0}(t)-e^{\lambda t} I\right)^{-1} U(t)\right)$. Now from the invertibility of $S_{0}(t)-e^{\lambda t} I$ 
and the compactness of $\left(S_{0}(t)-e^{\lambda t} I\right)^{-1} U(t)$ we conclude that

$$
e^{\lambda t} \in \sigma\left(T_{w}(t)\right) \Rightarrow e^{\lambda t} \in P \sigma\left(T_{w}(t)\right) .
$$

If $\lambda \in \mathbb{C}$ is such that $\operatorname{Re} \lambda \leqslant-\gamma x$ then $\lambda \in \sigma\left(A_{w}\right)$, according to Theorem 2.5. Now, using the spectral mapping results

$$
e^{t \sigma\left(A_{w}\right)} \subseteq \sigma\left(T_{w}(t)\right) \quad \text { and } \quad P \sigma\left(T_{w}(t)\right) \subseteq e^{t P \sigma\left(A_{w}\right)} \cup\{0\}
$$

(see e.g. [16]) we conclude that

$$
\sigma\left(T_{w}(t)\right)=\{0\} \cup\left\{e^{t \lambda} \mid \operatorname{Re} \lambda \leqslant-\gamma x \vee \lambda \in P \sigma\left(A_{w}\right) \cap \Omega\right\}
$$

for all $t \geqslant 0$. In Sect. 2 we found the following decomposition of $X$.

$$
X=N\left(A_{w}\right) \oplus R\left(A_{w}\right) .
$$

Let $\hat{T}_{w}(t)$ be the restriction of $T_{w}(t)$ to $R\left(A_{w}\right)$. Then $\hat{T}_{w}(t)$ defines a strongly continuous semigroup on $R\left(A_{w}\right)$ having infinitesimal generator $\hat{A}_{w}$, where $\hat{A}_{w}$ is the restriction of $A_{w}$ to $R\left(A_{w}\right)$. It follows that $\sigma\left(\hat{A}_{w}\right)=\sigma\left(A_{w}\right) \backslash\{0\}$ and $\sigma\left(\hat{T}_{w}(t)\right)=$ $\sigma\left(T_{w}(t)\right) \backslash\{1\}$. Therefore $r\left(\hat{T}_{w}(t)\right)=e^{-\nu t}, t \geqslant 0$. Now a result of Hale $([7$, Lemma 7.4.2]) yields: for all $\eta>0$ there exists a constant $K(\eta)>0$ such that for all $\phi \in R\left(A_{w}\right)$ and $t \geqslant 0$ :

$$
\left\|\hat{T}_{w}(t) \phi\right\| \leqslant K(\eta) e^{-(\nu-\eta) t}\|\phi\| .
$$

Let $\phi \in X$, then $T_{w}(t) \phi=T_{w}(t)(P \phi+(I-P) \phi)=P \phi+\hat{T}_{w}(t)(I-P) \phi$. Hence

$$
\left\|T_{w}(t) \phi-P \phi\right\| \leqslant K(\eta) e^{-(\nu-\eta) t}\|(I-P) \phi\| \leqslant K(\eta) e^{-(\nu-\eta) t}\|\phi\| .
$$

We can state our main result now.

Corollary 3.5. Let $n(t, s)$ be the solution of (1.7), then

$$
\lim _{t \rightarrow \infty} n(t, \cdot)=\int_{0}^{c+w} \phi(s) d \Psi_{w}(s) \cdot 1
$$

in the sup-norm.

Remark 3.2: Notice that $T_{w}(t) l=1, t \geqslant 0$. A semigroup satisfying this property is sometimes called a Markov-semigroup. (See e.g. [3]).

\section{The guzzler limit}

As we did mention in the Introduction Metz and van Batenburg [14] started from a more general model than we did. The forward equation (1.1) was obtained from this general model by a limit transition accounting for very small handling times. They even went one step further by letting the prey weight $w$ tend to zero while letting prey density $x$ tend to $\infty$, in order to arrive at a rather simple equation. Note that it is necessary to let simultaneously increase the prey density $x$. (Otherwise there would be nothing left to eat.) In this section we shall give a rigorous justification of this limit transition. We assume that

$$
\xi=x w
$$


and

$$
c^{*}=c+w
$$

remain constant. It follows from the interpretation that $\xi$ stands for the total prey biomass in the predator's environment, and that $c^{*}$ denotes the maximum gut content. Although this is not explicit in our notation, the prey capture rate may depend on $w$. We assume that

$$
g(s) \rightarrow g_{0}(s), \quad w \rightarrow 0,
$$

uniformly in $s \in\left[0, c^{*}\right]$, and $g_{0}$ is Lipschitz continuous.

A formal Taylor expansion of the backward Eq. (1.4) around $w=0$, neglecting higher order terms yields

$$
\frac{\partial n}{\partial t}(s, t) \approx-s \frac{\partial n}{\partial s}(s, t)+x w g(s) \frac{\partial n}{\partial s}(s, t) .
$$

If we let $w \rightarrow 0$, we obtain

$$
\frac{\partial n}{\partial t}(s, t)=\left(\xi g_{0}(s)-s\right) \frac{\partial n}{\partial s}(s, t),
$$

where we have used (4.1) and (4.3). We call (4.4) the limiting backward equation. The associated forward equation is given by

$$
\frac{\partial p}{\partial t}(s, t)=-\frac{\partial}{\partial s}\left(\left(\xi g_{0}(s)-s\right) p(s, t)\right),
$$

supplied with the boundary conditions

$$
p(s, t)=0, \quad s \leqslant 0 \quad \text { and } \quad s \geqslant c^{*} .
$$

Remark 4.1: We have to add the boundary condition $p(s, t)=0$ if $s \leqslant 0$, which is not present for $w>0$, because the characteristic curves associated with (4.5a) are directing inwards at $s=0$.

An important feature of (4.4) and (4.5a) is the absence of "jump terms": the catch of prey has become a deterministic process. The mantid's satiation now obeys the ordinary differential equation

$$
\frac{d s}{d t}=\xi g_{0}(s)-s
$$

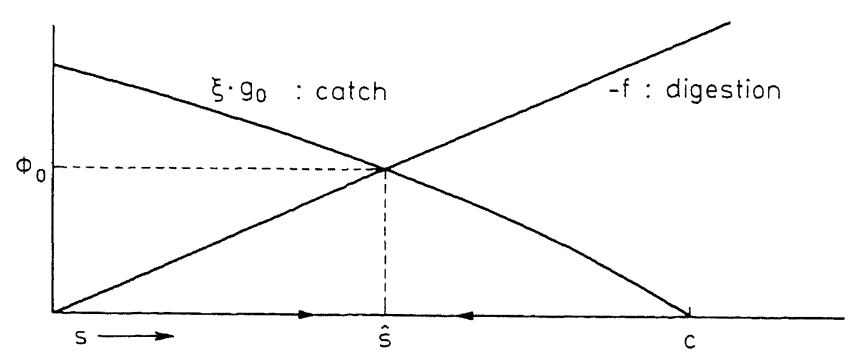

Fig. 1. In the guzzler limit prey catch $\xi \cdot g_{0}$ has become a deterministic process. The satiation $s$ of the predator tends to $\hat{s}$, and the functional response tends to $\Phi_{0}$ (see Sect. 7) 
Observe that (4.6) has a unique solution because the expression at the right-hand side defines a Lipschitz continuous function because of (4.3). (4.4) is written abstractly as

$$
\frac{d n}{d t}=A_{0} n
$$

where $A_{0}$ is given by

$$
\left(A_{0} \psi\right)(s)=-\left(s-\xi g_{0}(s)\right) \frac{d \psi}{d s}
$$

having a domain

$$
\begin{gathered}
D\left(A_{0}\right)=\{\psi \in X \mid \psi \text { is absolutely continuous and the function } \\
\left.s \mapsto\left(s-\xi g_{0}(s)\right) \frac{d \psi(s)}{d s} \text { is an element of } X\right\} .
\end{gathered}
$$

In this section we shall justify the formal limit transition by showing that (for identical initial data) solutions of $d n / d t=A_{w} n$, where $A_{w}$ is given by (1.8)-(1.9) converge to solutions of (4.7) if $w \rightarrow 0$. Let

$$
q(s)=\xi g_{0}(s)-s, \quad 0 \leqslant s \leqslant c^{*},
$$

and let $\hat{s}$ be the (unique) solution of $q(\hat{s})=0$. (Notice that assumption $(A)$ guarantees that $\hat{s}$ is uniquely determined.) Now let

$$
\begin{gathered}
Q_{l}(s)=\int_{0}^{s} \frac{d \sigma}{q(\sigma)}, \quad 0 \leqslant s<\hat{s}, \\
Q_{\imath}(s)=-\int_{s}^{c^{*}} \frac{d \sigma}{q(\sigma)}, \quad \hat{s}<s \leqslant s^{*} .
\end{gathered}
$$

Observe that $Q_{\ell}, Q_{\imath}$ are well-defined and $C^{1}$ on $[0, \hat{s})$ and $\left(\hat{s}, c^{*}\right]$ respectively. The solution of (4.4) supplied with the initial condition $n(s, 0)=\phi(s)$ is given by

$$
\begin{aligned}
& n(s, t)=\phi\left(Q_{\ell}^{-1}\left(t+Q_{\ell}(s)\right)\right), \quad 0 \leqslant s<\hat{s}, \\
& n(s, t)=\phi(\hat{s}), \quad s=\hat{s}, \\
& n(s, t)=\phi\left(Q_{\imath}^{-1}\left(t+Q_{t}(s)\right)\right), \quad \hat{s}<s \leqslant c^{*},
\end{aligned}
$$

where $Q_{\ell}^{-1}$ and $Q_{\imath}^{-1}$ denote the inverse functions of $Q_{\ell}$ and $Q_{\imath}$ respectively. It follows directly that the mapping $\phi \rightarrow n(\cdot, t)$, where $n(s, t)$ is given by $(4.11)$, defines a strongly continuous semigroup on $X$ which we denote with $T_{0}(t)$. The following result is straightforward.

Theorem 4.1. $\lim _{t \rightarrow \infty} T_{0}(t) \phi=\phi(\hat{s}) \cdot 1, \phi \in X$.

Theorem 4.2. For all $\phi \in X$ we have $\lim _{w \rightarrow \infty} T_{w}(t) \phi=T_{0}(t) \phi$, and this limit is uniform for $t$ in bounded intervals.

Proof: We use a Trotter-Kato type theorem to establish this result. Let $D$ be the subspace of $X$ consisting of $C^{1}$-functions. First we shall prove that for every $f \in D$ there exists an element $\psi \in D$ such that $\left(I-A_{0}\right) \psi=f$. Let $Q(s):=Q_{\ell}(s)$, 
$s<\hat{s}, Q(s):=Q_{\imath}(s), s>\hat{s}$. It follows immediately that

$$
\psi(s)=-e^{Q(s)} \int_{\hat{s}}^{s} \frac{f(\sigma) e^{-Q(\sigma)}}{q(\sigma)} d \sigma
$$

defines a solution of $\psi(s)-q(s) d \psi / d s=f(s)$. Suppose that $q(s)=\alpha(\hat{s}-s)$, then

$$
\psi(s)=\frac{1}{\alpha|s-\hat{s}|^{p}} \int_{\hat{s}}^{s} \frac{f(\sigma)|\sigma-\hat{s}|^{p}}{(\sigma-\hat{s})} d \sigma,
$$

where $p=1 / \alpha$. If $f \in D$, then

$$
f(\sigma)=f(\hat{s})+(\sigma-\hat{s}) f^{\prime}(\hat{s})+o(|\sigma-\hat{s}|)
$$

for $\sigma$ in a neighbourhood of $\hat{s}$. Substituting this in the expression for $\psi$, we find that for $s$ in a neighbourhood of $\hat{s}$

$$
\psi(s)=f(\hat{s})+\frac{f^{\prime}(\hat{s})}{\alpha(p+1)}(s-\hat{s})+o(|s-\hat{s}|) .
$$

Thus for this special choice of $q$, it follows that $D \subseteq\left(I-A_{0}\right) D$. The same result can be proved for arbitrary $q$ obtained from (4.9). (Here we have used the Lipschitz-continuity of $g$.) Moreover, it follows that for all $\psi \in D$ we have $\lim _{w \rightarrow 0}\left\|A_{w} \psi-A_{0} \psi\right\|=0$, where we have used (4.3). Now the Trotter-Kato theorem (See [16, Chapter 3, Theorem 4.5]) yields the result.

A straightforward computation shows that $\sigma\left(A_{0}\right)=\{\lambda \in \mathbb{C} \mid \operatorname{Re} \lambda \leqslant 0\}$. The eigenvector of $A_{0}$ corresponding to the eigenvalue $\lambda=0$ is 1 . The adjoint operator $A_{0}^{*}$ has the eigenvector

$$
\Psi_{0}(s)=-H(\hat{s}-s)
$$

corresponding to the eigenvalue $\lambda=0$. Here $H$ denotes the Heaviside function, i.e. $H(x)=0, x<0, H(x)=1, x>0$.

\section{The forward equation}

In Sect. 3 we solved the backward equation (1.4). The solutions were seen to be represented by a strongly continuous semigroup $T_{w}(t)$. Solutions of the forward equation (1.1) are to be regarded as linear functionals on the space $X$ of continuous functions and they are called weak $*$ solutions (cf. [1]). The idea becomes more clear if we integrate (1.1a) from $s$ to $c+w$. We obtain

$$
\frac{\partial P(s, t)}{\partial t}=s \frac{\partial}{\partial s}(P(s, t))-x \int_{s-w}^{s} g(\sigma) d P(\sigma, t),
$$

where $P(s, t)=-\int_{s}^{c+w} p(\sigma, t)$. Now $P(\cdot, t)$ is a bounded variation function normalized by the condition

$$
P(s, t)=0, \quad s \geqslant c+w
$$


i.e. $P(\cdot, t) \in X^{*}, t \geqslant 0$, where $X^{*}$ is given by (2.27). Equation (5.1) has to be supplemented with the initial condition

$$
P(s, 0)=P_{0}(s),
$$

where $P_{0}(s)=-\int_{s}^{c+w} p_{0}(\sigma) d \sigma$, and $p_{0}(\cdot)$ is given by $(1.1 \mathrm{c}) .(5.1)-(5.2)$ can be rewritten as

$$
\frac{d P}{d t}=A_{w}^{*} P, \quad P(0)=P_{0},
$$

where $A_{w}^{*}$ is given by (2.28)-(2.29). The solution of (5.3) which we denote by $P\left(s, t ; P_{0}\right)$ is characterized by the following relation (see (1.6)):

$$
\int_{0}^{c+w} \phi(s) d P\left(s, t ; P_{0}\right)=\int_{0}^{c+w} n(s, t ; \phi) d P_{0}(s), \quad \phi \in X,
$$

where $n(\cdot, t ; \phi)=T_{w}(t) \phi$ is the solution of the backward equation (1.4).

Up till now we did not mention what topology $X^{*}$ is endowed with. The sense in which solutions of the integrated forward equation (5.1) should be interpreted, namely being linear functionals on the space of continuous functions $X$, yields that we should work with the weak $*$ topology on $X^{*}$. This topology is characterized if we define what convergence of a sequence in $X^{*}$ means: let $\left\{\Psi_{n}\right\}_{n \in \mathbb{N}}$ be a sequence in $X^{*}$, and let $\Psi \in X^{*}$. We say that $\Psi_{n} \rightarrow \Psi$ in the weak * topology of $X^{*}$ if for all $\phi \in X$

$$
\int_{0}^{c+w} \phi(s) d \Psi_{n}(s) \rightarrow \int_{0}^{c+w} \phi(s) d \Psi(s), \quad n \rightarrow \infty .
$$

(See e.g. $[1,18]$.)

Now let us return to our forward equation (5.1). Condition (1.2) can be rewritten as

$$
\int_{0}^{c+w} d P_{0}(s)=1
$$

If $P_{0}$ satisfies (5.5), then so does the solution $P\left(\cdot, t ; P_{0}\right)$ of $(5.3)$ for all $t \geqslant 0$. (See (1.2)). Now we shall reformulate Theorem 3.4 in terms of $P\left(\cdot, t ; P_{0}\right)$. Let $\Psi_{w}$ be the eigenvector of $A_{w}^{*}$ associated with the dominant eigenvalue 0 . (See Sect. 2.)

Corollary 5.1. Let $\nu$ be given by (3.8) and let $\eta>0$ be arbitrary. If $P_{0}$ satisfies (5.5) then

$$
P\left(\cdot, t ; P_{0}\right)=\Psi_{w}+\mathcal{O}\left(e^{-(\nu-\eta) t}\right), \quad t \rightarrow \infty
$$

in the weak * topology of $X^{*}$.

We define the family of operators $T_{w}^{*}(t)$ by

$$
T_{w}^{*}(t) P_{0}=P\left(\cdot, t ; P_{0}\right) \text {. }
$$

Then $T_{w}^{*}(t)$ is the adjoint operator of $T_{w}(t)$ for all $t \geqslant 0$, and $T_{w}^{*}(t)$ defines a weak $*$ semigroup on $X^{*}$ (see [1]), i.e.

(i) $T_{w}^{*}\left(t_{1}\right) T_{w}^{*}\left(t_{2}\right)=T_{w}^{*}\left(t_{1}+t_{2}\right)$, 
(ii) $T_{w}^{*}(0)=I$,

(iii) $\lim _{t \downarrow 0}\left\langle T_{w}^{*}(t) \Psi, \phi\right\rangle=\langle\Psi, \phi\rangle$, for all $\phi \in X, \Psi \in X^{*}$.

$A_{w}^{*}$ is the weak $*$ infinitesimal generator of the weak $*$ semigroup $T_{w}(t)$, i.e.

$$
\lim _{t \downarrow 0}\left\langle\frac{T_{w}^{*}(t)-I}{t} \Psi, \phi\right\rangle=\left\langle A_{w}^{*} \Psi, \phi\right\rangle, \text { for all } \phi \in X \text { and } \Psi \in \mathscr{D}\left(A_{w}^{*}\right) \text {. }
$$

More details can be found in the book of Butzer and Behrens [1].

Also Theorem 4.1, characterizing the asymptotic behaviour of the limiting backward equation $d n / d t=A_{0} n$, can be reformulated in terms of bounded variation functions. As above we can associate a weak $*$ semigroup $T_{0}^{*}(t)$ with the solutions of the integrated limiting forward equation (4.5).

Corollary 5.2. Let $\Psi_{0}$ be given by (4.12). If $P_{0}$ satisfies (5.5) then $\lim _{t \rightarrow \infty} T_{0}^{*}(t) P_{0}=$ $\Psi_{0}$, with respect to the weak $*$ topology of $X^{*}$.

This means that solutions of the non-integrated limiting forward equation (4.5) converge in distribution-sense to the delta function $\delta(s-\hat{s})$.

From Theorem 4.2 it can be easily seen what happens to solutions of the forward equation (1.1) if the prey weights $w$ become very small.

Corollary 5.3. Let $P_{0}$ satisfy (5.5). Then $\lim _{w \rightarrow 0} T_{w}^{*}(t) P_{0}=T_{0}^{*}(t) P_{0}$ in the weak $*$ topology of $X^{*}$, and this limit is uniform for $t$ in bounded intervals.

So far, it is not clear whether the result of Corollary 5.3 is also valid for $t \rightarrow \infty$. If this is true then it follows from the Corollaries 5.1 and 5.2 that $\Psi_{w} \rightarrow \Psi_{0}$ if $w \rightarrow 0$. This can indeed be proved.

Theorem 5.4. $\lim _{w \rightarrow 0}\left\langle\Psi_{w}, \phi\right\rangle=\left\langle\Psi_{0}, \phi\right\rangle$ for all $\phi \in X$.

Proof: Let $t>0$ be fixed. Then $T_{w}^{*}(t) \Psi_{w}=\Psi_{w}$. If $\phi \in X,\|\phi\| \leqslant 1$, then

$$
\left|\left\langle\Psi_{w}, \phi\right\rangle\right|=\left|\int_{0}^{c+w} \phi(s) d \Psi_{w}(s)\right| \leqslant\left|\int_{0}^{c+w} d \Psi_{w}(s)\right|=1,
$$

where we have used that $\Psi_{w}$ is increasing, $\Psi_{w}(0)=-1, \Psi_{w}(c+w)=0$. (See Sect. 2.) Therefore $\Psi_{w}$ is an element of the closed unit ball in $X^{*}$, for all $w>0$. Alaoglu's theorem (see [18, Theorem III.10.2]) states that this unit ball is weak * compact. As a consequence the set $\left\{\Psi_{w} \mid w>0\right\}$ has at least one limit point within the closed unit ball. Let $\chi$ be such a limit point. Then there exists a sequence $\left\{w_{k}\right\}_{k \in N}$ such that $w_{k} \rightarrow 0$ if $k \rightarrow \infty$ and $\Psi_{w_{k}} \rightarrow \chi, k \rightarrow \infty$ with respect to the weak $*$ topology of $X^{*}$. Now

$$
\begin{aligned}
\left|\left\langle T_{0}^{*}(t) \chi-\chi, \phi\right\rangle\right| & =\left|\left\langle T_{0}^{*}(t) \chi-T_{w_{k}}^{*}(t) \chi+T_{w_{k}}^{*}(t) \chi-T_{w_{k}}^{*}(t) \Psi_{w_{k}}+\Psi_{w_{k}}-\chi, \phi\right\rangle\right| \\
& =\left|\left\langle\chi, T_{0}(t) \phi-T_{w_{k}}(t) \phi\right\rangle+\left\langle\chi-\Psi_{w_{k}}, T_{w_{k}}(t) \phi\right\rangle+\left\langle\Psi_{w_{k}}-\chi, \phi\right\rangle\right| \\
& \leqslant\left|\left\langle\Psi_{w_{k}}, T_{0}(t) \phi-T_{w_{k}}(t) \phi\right\rangle\right|+\left|\left\langle\chi-\Psi_{w_{k}}, T_{0}(t) \phi-\phi\right\rangle\right| \\
& \leqslant\left\|T_{0}(t) \phi-T_{w_{k}}(t) \phi\right\|+\left|\left\langle\chi-\Psi_{w_{k}}, T_{0}(t) \phi-\phi\right)\right| .
\end{aligned}
$$

If we let $k \rightarrow \infty$, then this expression tends to zero, from which we conclude

$$
T_{0}^{*}(t) \chi=\chi .
$$


Thus $\left\langle\chi, T_{0}(t) \phi\right\rangle=\langle\chi, \phi\rangle$ for all $\phi \in X$, and this relation is valid for all $t \geqslant 0$. Now letting $t \rightarrow \infty$ and using Theorem 4.1 we find

$$
\langle\chi, \phi\rangle=\phi(\hat{s})\langle\chi, 1\rangle=\lim _{k \rightarrow \infty} \phi(\hat{s})\left\langle\Psi_{w_{k}}, 1\right\rangle=\phi(\hat{s}) .
$$

Therefore $\chi=\Psi_{0}$, and this result is independent of the choice of the sequence $\left\{w_{k}\right\}_{k \in \mathbb{N}}$. This yields the result.

\section{The inhomogeneous equation}

Here we shall study the inhomogeneous equation (1.11) which we first rewrite in terms of bounded variation functions. Let

$$
Z(s, t)=\int_{s}^{c+w} z(\sigma, t) d \sigma .
$$

Integration of (1.11a) from $s$ to $c+w$ yields that $Z$ must obey

$$
\frac{\partial Z}{\partial t}=s \frac{\partial Z}{\partial s}-x \int_{s-w}^{s} g(\sigma) d Z(\sigma, t)+H(s, t)
$$

where $H(t)=H(t, \cdot)$ is the bounded variation function given by

$H(t, s)=-x \int_{s-w}^{c+w} g(\sigma) d P(\sigma, t)+x \int_{0}^{c+w} g(\sigma) d P(\sigma, t) \cdot \int_{s}^{c+w} d P(\sigma, t)$,

where $P(s, t)$ is the solution of $(5.1)-(5.2)$, i.e. $P(s, t)=\left(T_{w}^{*}(t) P_{0}\right)(s)$, and $P_{0}$ satisfies (5.5). Obviously

$$
\langle H(t), 1\rangle=0 .
$$

From $(1.11 \mathrm{~b})-(1.11 \mathrm{c})$ it follows that $(6.1)$ has to be supplied with the boundary and initial conditions

$$
\begin{array}{ll}
Z(s, t)=0, & s \geqslant c+w \\
Z(s, 0)=0, & 0 \leqslant s \leqslant c+w .
\end{array}
$$

Now we can rewrite (6.1), (6.4) as an abstract Cauchy problem.

$$
\frac{d Z}{d t}=A_{w}^{*} Z+H(t), \quad Z(0)=0
$$

Taking the innerproduct of (6.5) with an arbitrary element $\phi \in \mathscr{D}\left(A_{w}\right)$ we find the ordinary differential equation:

$$
\frac{d}{d t}\langle Z(t), \phi\rangle=\left\langle Z(t), A_{w} \phi\right\rangle+\langle H(t), \phi\rangle, \quad\langle Z(0), \phi\rangle=0 .
$$

The solution of this equation is given by

$$
\langle Z(t), \phi\rangle=\int_{0}^{t}\left\langle H(\tau), T_{w}(t-\tau) \phi\right\rangle d \tau
$$


The remainder of this section is devoted to the study of the large-time behaviour of this solution. We need the following result. Let the bounded variation function $H_{w}$ be given by

$$
H_{w}(s)=-x \int_{s-w}^{c+w} g(\sigma) d \Psi_{w}(\sigma)+x \int_{0}^{c+w} g(\sigma) d \Psi_{w}(\sigma) \cdot \int_{s}^{c+w} d \Psi_{w}(\sigma) .
$$

Lemma 6.1. Let $\nu$ be given by (3.8). For all $\eta>0$ there exists a constant $L(\eta)>0$ such that for all $\phi \in X$

$$
\left|\int_{0}^{c+w} \phi(s) d H(s, t)-\int_{0}^{c+w} \phi(s) d H_{w}(s)\right| \leqslant L(\eta) e^{-(\nu-\eta) t}\|\phi\| .
$$

Proof:

$$
\begin{aligned}
\mid \int_{0}^{c+\dot{w}} & \phi(s) d H(s, t)-\int_{0}^{c+w} \phi(s) d H_{w}(s) \mid \\
= & \mid \int_{0}^{c+w} \phi(s) \cdot\left\{x g(s-w) d P(s-w, t)-x d P(s, t) \cdot \int_{0}^{c+w} g(\sigma) d P(\sigma, t)\right\} \\
& -\int_{0}^{c+w} \phi(s)\left\{x g(s-w) d \Psi_{w}(s-w)-x d \Psi_{w}(s) \cdot \int_{0}^{c+w} g(\sigma) d \Psi_{w}(\sigma)\right\} \mid .
\end{aligned}
$$

Corollary 5.1 states that for every $\phi \in X$

$$
\left|\int_{0}^{c+w} \phi(s) d P(s, t)-\int_{0}^{c+w} \phi(s) d \Psi_{w}(s)\right| \leqslant K(\eta) e^{-(\nu-\eta) t}\|\phi\|,
$$

for some positive constant $K(\eta)$. This and the continuity of $g$ yield the result.

Theorem 6.2. Let for all $\phi \in X,\langle Z(t), \phi\rangle$ be defined by (6.7). Then

$$
\lim _{t \rightarrow \infty}\langle Z(t), \phi\rangle=\left\langle H_{w},-A_{w}^{-1}(I-P) \phi\right\rangle,
$$

where $P$ is the projection on $N\left(A_{w}\right)$ given by (2.32).

Proof: Let $\phi \in X$ and $\psi$ its projection on $R\left(A_{w}\right)$, i.e. $\psi=(I-P) \phi$. Then

$$
\langle Z(t), \phi\rangle=\int_{0}^{t}\left\langle H(\tau), T_{w}(t-\tau)(P \phi+\psi)\right\rangle d \tau=\int_{0}^{t}\left\langle H(\tau), T_{w}(t-\tau) \psi\right\rangle d \tau,
$$

where we have used that $\left\langle H(\tau), T_{w}(t-\tau) P \phi\right\rangle=\left\langle H(\tau),\left\langle\Psi_{w}, \phi\right\rangle \cdot 1\right\rangle=0$, because of (6.3). Hence

$$
\langle Z(t), \phi\rangle=\int_{0}^{t}\left\langle H_{w}, T_{w}(t-\tau) \psi\right\rangle d \tau+\int_{0}^{t}\left\langle H(\tau)-H_{w}, T_{w}(t-\tau) \psi\right\rangle d \tau .
$$

Let $\eta>0$. Lemma 6.1 and Theorem 3.4 yield that

$$
\begin{aligned}
& \left|\int_{0}^{t}\left\langle H(\tau)-H_{w}, T_{w}(t-\tau) \psi\right\rangle d \tau\right| \leqslant \int_{0}^{t} L(\eta) e^{-(\nu-\eta) \tau}\left\|T_{w}(t-\tau) \psi\right\| d \tau \\
& \quad \leqslant K(\eta) L(\eta) \int_{0}^{t} e^{-(\nu-\eta) \tau} e^{-(\nu-\eta)(t-\tau)}\|\psi\| d \tau \\
& \quad=K(\eta) L(\eta) t e^{-(\nu-\eta) t}\|\psi\|,
\end{aligned}
$$


Finally

$$
\int_{0}^{t}\left\langle H_{w}, T_{w}(t-\tau) \psi\right\rangle d \tau=\left\langle H_{w}, \int_{0}^{t} T_{w}(\tau) \psi d \tau\right\rangle \rightarrow\left\langle H_{w},-A^{-1} \psi\right\rangle .
$$

if $t \rightarrow \infty$, where we have used a standard result from semigroup theory. (See e.g. [16].)

This proves the theorem.

We'shall give a more comprehensible reformulation of this result. Let $Z_{w} \in X^{*}$ be defined by

$$
\begin{aligned}
A_{w}^{*} Z_{w} & =-H_{w} \\
\left\langle Z_{w}, 1\right\rangle & =0 .
\end{aligned}
$$

(Existence of $Z_{w}$ is guaranteed by the Fredholm Alternative $\left(\left\langle H_{w}, 1\right\rangle=0\right)$. The second condition guarantees uniqueness.)

Let $Z(s, t)$ be the weak * solution of (6.1), defined by (6.7).

Corollary 6.3. $Z(s, t) \rightarrow Z_{w}(s), t \rightarrow \infty$ in the weak * topology of $X^{*}$.

Remark 6.1: It doesn't make sense to study the inhomogeneous equation that is obtained if one lets $w \rightarrow 0$ in (1.11). To understand this, one should remember that the solution $z$ of $(1.11)$ is needed to calculate the variance $\operatorname{var}(W)$ of prey catch $W$ per unit of time (see Remark 1.4). However, if $w \rightarrow 0$ then the catching process becomes deterministic, yielding that $\operatorname{var}(W)$ vanishes, and hence $Z_{w}(s) \rightarrow$ 0 if $w \rightarrow 0$.

\section{The functional response}

In this paper we define the functional response $\Phi_{w}(\xi)$ as the total weight of prey caught per unit of time per predator, where $\xi=x w$ is the density of prey weight in the mantid's environment.

Remark 7.1: Observe that $\Phi_{w}$ is a function of two independent variables, $\xi$ and $w$. One might also choose $x$ and $w$ or $\xi$ and $x$. However in practical cases, $w$ can be chosen a constant and the functional reponse is a function of $\xi$ only. In many cases biologists prefer to work with $x$ instead of $\xi$. In our case $\xi$ is a better choice because later on, we shall take the limit, $w \rightarrow 0, x \rightarrow \infty$ such that $\xi=x w$ remains constant, and we want to examine what happens to the functional response in this case.

$\Phi_{w}(\xi)$ can be calculated from

$$
\Phi_{w}(\xi)=\xi \int_{0}^{c+w} g(s) d \Psi_{w}(s)
$$

where $\Psi_{w}$ is the (positive) eigenvector of $A_{w}^{*}$ corresponding to the dominant eigenvalue $\lambda=0$, normalized by the condition

$$
\left\langle\Psi_{w}, 1\right\rangle=\int_{0}^{c+w} d \Psi_{w}(s)=1 .
$$


In experiments, $\Phi_{w}(\xi)$ is found to be increasing and concave and to have a finite limit for $\xi \rightarrow \infty$. We have tried to prove these properties by means of analytical methods, but we have not succeeded so far. However, if we let $w$ tend to zero, keeping $\xi=x w$ and $c^{*}=c+w$ constant, then we find that $\Psi_{0}(s)=-H(s-\hat{s})$. From (7.1) we find that for $w=0$ the functional response $\Phi_{0}(\xi)$ is given by

$$
\Phi_{0}(\xi)=\xi g_{0}(\hat{s})=\hat{s}
$$

where $\hat{s}$ is the unique solution of

$$
\xi g_{0}(s)=s \quad \text { (See Fig. 1). }
$$

It is clear that $\hat{s}$ depends on $\xi$, and a straightforward computation shows that $\Phi_{0}(\xi)$ is increasing. Moreover $\lim _{\xi \rightarrow \infty} \Phi_{0}(\xi)=c^{*}=c$.

Example: If $g(s)$ is linear, $g(s)=\gamma(1-s / c)^{+}$, the ${ }^{+}$meaning that negative values are replaced by zero, then $g_{0}(s)=\gamma\left(1-s / c^{*}\right)^{+}$and $\Phi_{0}(\xi)=\hat{s}=\gamma c^{*} \xi /\left(c^{*}+\gamma \xi\right)$.

The usefulness of $\Phi_{0}(\xi)$ is demonstrated by the following result, which says that $\Phi_{0}(\xi)$ approximates $\Phi_{w}(\xi)$ for small $w$.

Theorem 7.1. For all $\xi>0$ we have

$$
\lim _{w \rightarrow 0} \Phi_{w}(\xi)=\Phi_{0}(\xi)
$$

Proof: (7.1) says that $\Phi_{w}(\xi)=\xi\left\langle\Psi_{w}, g\right\rangle$. Hence

$$
\begin{aligned}
\left|\Phi_{w}(\xi)-\Phi_{0}(\xi)\right| & =\xi\left|\left\langle\Psi_{w}, g\right\rangle-\left\langle\Psi_{0}, g_{0}\right\rangle\right|=\xi\left|\left\langle\Psi_{w}, g-g_{0}\right\rangle+\left\langle\Psi_{w}-\Psi_{0}, g_{0}\right\rangle\right| \\
& \leqslant \xi\left\|g-g_{0}\right\|+\xi\left|\left\langle\Psi_{w}-\Psi_{0}, g_{0}\right\rangle\right|
\end{aligned}
$$

and this tends to zero if $w \rightarrow 0$ because of (4.3) and Theorem 5.4.

Remark 7.2: It follows from the proof of Theorem 7.1 that

in the sup-norm.

$$
\frac{\Phi_{w}(\xi)}{\xi} \rightarrow \frac{\Phi_{0}(\xi)}{\xi}
$$

We were able to compute $\Phi_{w}(\xi)$ for a special case, namely $c \leqslant w$. Biologically, this means that the predator's gut can contain at most two preys. After consuming a prey, the predator will not show prey catching behaviour until (part of) the previous meal is digested. Now let

$$
\psi_{w}=\frac{d}{d s} \Psi_{w}(s)
$$

Then $s \rightarrow s \psi_{w}(s)$ defines an $L_{1}$-function. (2.30) yields

$$
\frac{d}{d s}\left(s \psi_{w}(s)\right)-x g(s) \psi_{w}(s)+x g(s-w) \psi_{w}(s-w)=0 .
$$

If we substitute $\theta(s)=s \psi_{w}(s) / E(s)$ where $E$ is given by (2.2) in the first two terms we obtain

$$
\frac{d \theta}{d s}=-x g(s-w) \cdot \frac{1}{E(s)} \psi_{w}(s-w) .
$$


Integration from $s$ to $c+w$ and the fact that $\psi_{w}(c+w)=0$ yield

$$
\theta(s)=x \int_{s}^{c+w} g(\sigma-w) \frac{1}{E(\sigma)} \psi_{w}(\sigma-w) d \sigma
$$

and we obtain

$$
\psi_{w}(s)=\frac{x E(s)}{s} \int_{s}^{c+w} \frac{g(\sigma-w)}{E(\sigma)} \psi_{w}(\sigma-w) d \sigma .
$$

From (7.2) we conclude that

$$
\int_{0}^{c+w} \psi_{w}(s) d s=1
$$

Observe that (7.4)-(7.6) are also valid if $c>w$. Now the functional response can be computed from

$$
\Phi_{w}(\xi)=\xi \int_{0}^{c+w} g(\sigma) \psi_{w}(\sigma) d \sigma
$$

With respect to $\psi_{w}(s)$ we can prove the following: $\psi_{w}(s)$ is continuous on $(0, c+w], \psi_{w}(s)=\mathscr{O}\left(s^{\gamma x-1}\right), s \downarrow 0, \psi_{w} \in L_{1}[0, c+w], \psi_{w}(s) \geqslant 0$ a.e. on $[0, c+w]$.

Now let us assume that $c \leqslant w$, then $E(s)=1, c \leqslant s \leqslant c+w$. From (7.5) we find that $\psi_{w}(s)=N . E(s) / s$ if $0 \leqslant s \leqslant w$ for some constant $N$. For $w \leqslant s \leqslant c+w$ we have

$$
\psi_{w}(s)=\frac{x}{s} \int_{s}^{c+w} g(\sigma-w) N \frac{E(\sigma-w)}{\sigma-w} d \sigma=\frac{N}{s}(1-E(s-w)) .
$$

Now $N$ should be computed from (7.6).

$$
\begin{aligned}
1 & =N\left\{\int_{0}^{w} \frac{E(s)}{s} d s+\int_{w}^{c+w} \frac{1-E(s-w)}{s} d s\right\} \\
& =N\left\{\int_{c}^{c+w} \frac{d s}{s}+\int_{0}^{c} E(s)\left(\frac{1}{s}-\frac{1}{s+w}\right) d s\right\} \\
& =N\left\{\log \frac{c+w}{c}+w \int_{0}^{c} \frac{E(s)}{s(s+w)} d s\right\} .
\end{aligned}
$$

Now

$$
\Phi_{w}(\xi)=\xi \int_{0}^{c+w} g(s) \psi_{w}(s) d s=\xi N \int_{0}^{c} g(s) \frac{E(s)}{s} d s=w N(1-E(0))=w N
$$

if $\xi>0$, and $\Phi_{w}(\xi)=0$ if $\xi=0$.

Thus we have proved the following result.

Theorem 7.2. If $c \leqslant w$ then

$$
\begin{aligned}
& \Phi_{w}(\xi)=0 \quad \text { if } \xi=0 \\
& \Phi_{w}(\xi)=w\left(\log \left(\frac{c+w}{c}\right)+w \int_{0}^{c} \frac{E(s)}{s(s+w)} d s\right)^{-1} \text { if } \xi>0 .
\end{aligned}
$$


Observe that $\Phi_{w}(\xi)$ given by (7.8) is increasing and concave. Moreover

$$
\lim _{\xi \rightarrow \infty} \Phi_{w}(\xi)=w / \log \left(\frac{c+w}{c}\right) .
$$

Remark 7.3: From probabilistic considerations it follows that (7.9) is also valid if $c>w$. (See [15].)

Acknowledgement. I would like to thank Odo Diekmann for some valuable suggestions. Especially I would like to thank Hans Metz for many stimulating and inspiring discussions.

\section{Appendix}

In this appendix we shall prove that the adjoint operator $A_{w}^{*}$ of $A_{w}$ is given by

$$
\left(A_{w}^{*} \Psi\right)(s)=s \frac{d \Psi}{d s}-x \int_{s-w}^{s} g(\sigma) d \Psi(\sigma)
$$

having a domain

$$
\mathscr{D}\left(A_{\mathrm{w}}^{*}\right)=\left\{\Psi \in X^{*} \mid \Psi \text { is absolutely continuous and } s \rightarrow s \frac{d \Psi}{d s} \in X^{*}\right\} .
$$

Let $\lambda \in \mathbb{R}, \lambda>0$ be arbitrary. Then

$$
\mathscr{D}\left(A_{w}^{*}\right)=R\left(\left(\left(\lambda I-A_{w}\right)^{-1}\right)^{*}\right) .
$$

Theorem 2.1 says that $\lambda \psi-A_{w^{\prime}} \psi=h$ if and only if $\psi-\hat{T}_{\lambda} \psi=U_{\lambda} h$. Where $\hat{T}_{\lambda}$ and $U_{\lambda}$ are given by (2.9) and (2.10) respectively. Let $X^{*}$ be given by (2.27).

\section{Lemma A.1.}

$$
\left(U_{\lambda}^{*} F\right)(s)=-\int_{s}^{c+w} \sigma^{\lambda-1} E(\sigma)\left(\int_{\sigma}^{c+w} \frac{d F(\eta)}{\eta^{\lambda} E(\eta)}\right) d \sigma, \text { for all } F \in X^{*} .
$$

Proof: Let $\phi \in X$ and $F \in X^{*}$. Then

$$
\begin{aligned}
\left\langle F, U_{\lambda} \phi\right\rangle & =\int_{0}^{c+w}\left(U_{\lambda} \phi\right)(s) d F(s) \\
& =\int_{0}^{c+w} \frac{1}{s^{\lambda} E(s)}\left\{\int_{0}^{s} \sigma^{\lambda-1} E(\sigma) \phi(\sigma) d \sigma\right\} d F(s) \\
& =\int_{0}^{c+w} \frac{1}{s^{p} \tilde{E}(s)}\left\{\int_{0}^{s} \sigma^{p-1} \tilde{E}(\sigma) \phi(\sigma) d \sigma\right\} d F(s)
\end{aligned}
$$

where we have used (2.3) and where $p=\lambda+\gamma x$. Because this integral is absolutely convergent, we can apply Fubini's theorem and change order of integration.

$$
\begin{aligned}
\left\langle F, U_{\lambda} \phi\right\rangle & =\int_{0}^{c+w} \sigma^{\lambda-1} E(\sigma) \phi(\sigma)\left\{\int_{\sigma}^{c+w} \frac{d F(s)}{s^{\lambda} E(s)}\right\} d \sigma, \\
& =\langle G, \phi\rangle,
\end{aligned}
$$

where

$$
G(s)=-\int_{s}^{c+w} \sigma^{\lambda-1} E(\sigma)\left(\int_{\sigma}^{c+w} \frac{d F(\eta)}{\eta^{\lambda} E(\eta)}\right) d \sigma .
$$

We also have $\left\langle F, U_{\lambda} \phi\right\rangle=\left\langle U_{\lambda}^{*} F, \phi\right\rangle$ and therefore $U_{\lambda}^{*} F=G$, which yields the result. 
Obviously $\left(\lambda I-A_{w}\right)^{-1}=\left(I-\hat{T}_{\lambda}\right)^{-1} U_{\lambda}$, hence

$$
\left(\left(\lambda I-A_{w}\right)^{-1}\right)^{*}=U_{\lambda}^{*}\left(I-T_{\lambda}^{*}\right)^{-1} .
$$

From (3) and (4) it is clear that

$$
\mathscr{D}\left(A_{w}^{*}\right)=R\left(U_{\lambda}^{*}\right) .
$$

Theorem A.2. $\mathscr{D}\left(A_{w}^{*}\right)=V={ }^{\operatorname{def}}\left\{\Psi \in X^{*} \mid \Psi\right.$ is absolutely continuous and $s \mapsto s d \Psi(s) / d s$ is an element of $\left.X^{*}\right\}$.

Proof: (i) Suppose $\Psi \in \mathscr{D}\left(A_{\mathrm{w}}^{*}\right)$, then $\Psi \in R\left(U_{\lambda}^{*}\right)$. Let $F \in X^{*}$ be such that $U_{\lambda}^{*} F=\Psi$. It follows from Lemma A.1 that

$$
\Psi(s)=-\int_{s}^{c+w} \sigma^{\lambda-1} E(\sigma)\left(\int_{\sigma}^{c+w} \frac{d F(\eta)}{\eta^{\lambda} E(\eta)}\right) d \sigma .
$$

A straightforward computation shows that $\Psi$ is absolutely continuous on $[\varepsilon, c+w]$ for every $\varepsilon>0$. Moreover, using Fubini's theorem it follows directly that $\Psi$ is continuous on $[0, c+w]$. This yields that $\Psi$ is absolutely continuous on $[0, c+w]$. Obviously

$$
s \Psi^{\prime}(s)=s^{\lambda} E(s) \int_{s}^{c+w} \frac{d F(\eta)}{\eta^{\lambda} E(\eta)},
$$

and the right-hand expression defines an element of $X^{*}$. Thus $\mathscr{D}\left(A_{w}^{*}\right) \subset V$.

(ii) Now suppose that $\Psi \in V$. We shall prove that there exists an element $F \in X^{*}$ such that $U_{\lambda}^{*} F=\Psi$. Let

$$
F(s)=-s \frac{d \Psi}{d s}-\int_{s}^{c+n}(\lambda+x g(\sigma)) d \Psi(\sigma) .
$$

Clearly $F \in X^{*}$. From Lemma A.l we know that

$$
\left(U_{\lambda}^{*} F\right)(s)=-\int_{s}^{c+w} \sigma^{\lambda-1} E(\sigma)\left(\int_{\sigma}^{c+w} \frac{d F(\eta)}{\eta^{\lambda} E(\eta)}\right) d \sigma .
$$

First we compute the expression

$$
\begin{gathered}
\left.\int_{\sigma}^{c+w} \frac{d F(\eta)}{\eta^{\lambda} E(\eta)}=\frac{F(\eta)}{\eta^{\lambda} E(\eta)}\right]_{\sigma}^{c+w}+\int_{\sigma}^{c+w} F(\eta) \cdot\left(\frac{\lambda+x g(\eta)}{\eta^{\lambda+1} E(\eta)}\right) d \eta \\
=\frac{-F(\sigma)}{\sigma^{\lambda} E(\sigma)}+\int_{\sigma}^{c+w} F(\eta)\left(\frac{\lambda+x g(\eta)}{\eta^{\lambda+1} E(\eta)}\right) d \eta, \quad \text { if } \sigma>0 .
\end{gathered}
$$

If we substitute (6), we obtain

$$
\begin{aligned}
\int_{\sigma}^{c+w} \frac{d F(\eta)}{\eta^{\lambda} E(\eta)}= & \frac{\Psi^{\prime}(\sigma)}{\sigma^{\lambda-1} E(\sigma)}+\frac{1}{\sigma^{\lambda} E(\sigma)} \int_{\sigma}^{c+w}(\lambda+x g(\eta)) d \Psi(\eta) \\
& -\int_{\sigma}^{c+w} \eta \Psi^{\prime}(\eta) \cdot \frac{\lambda+x g(\eta)}{\eta^{\lambda+1} E(\eta)} d \eta \\
& -\int_{\sigma}^{c+w} \frac{\lambda+x g(\eta)}{\eta^{\lambda+1} E(\eta)}\left(\int_{\eta}^{c+w}(\lambda+x g(\xi)) d \Psi(\xi)\right) d \eta
\end{aligned}
$$

Again, Fubini's theorem says that we may change order of integration in the last expression at the right-hand side

$$
\begin{gathered}
\int_{\sigma}^{c+w} \frac{\lambda+x g(\eta)}{\eta^{\lambda+1} E(\eta)}\left(\int_{\eta}^{c+w}(\lambda+x g(\xi)) d \Psi(\xi)\right) d \eta=\int_{\sigma}^{c+w}(\lambda+x g(\xi))\left(\int_{\sigma}^{\xi} \frac{\lambda+x g(\eta)}{\eta^{\lambda+1} E(\eta)} d \eta\right) d \Psi(\xi) \\
=\frac{1}{\sigma^{\lambda} E(\sigma)} \int_{\sigma}^{c+w}(\lambda+x g(\xi)) d \Psi(\xi)-\int_{\sigma}^{c+w} \frac{\lambda+x g(\xi)}{\xi^{\lambda} E(\xi)} d \Psi(\xi) .
\end{gathered}
$$


Substitution in (7) yields

$$
\int_{\sigma}^{c+w} \frac{d F(\eta)}{\eta^{\lambda} E(\eta)}=\frac{\Psi^{\prime}(\sigma)}{\sigma^{\lambda-1} E(\sigma)}
$$

Consequently

$$
\left(U_{\lambda}^{*} F\right)(s)=-\int_{s}^{c+w} \sigma^{\lambda-1} E(\sigma) \frac{\Psi^{\prime}(\sigma)}{\sigma^{\lambda-1} E(\sigma)} d \sigma=\Psi(s) .
$$

Therefore $\Psi \in R\left(U_{\lambda}^{*}\right)=\mathscr{D}\left(A_{w}^{*}\right)$.

Now suppose that $\phi \in \mathscr{D}\left(A_{w^{\prime}}\right)$ and $\Psi \in \mathscr{D}\left(A_{w}^{*}\right)$. Then $\Psi$ is absolutely continuous. Let $\psi(s)=d \Psi / d s$, then $\psi$ is an $L_{1}$-function.

$$
\begin{aligned}
\left\langle\Psi, A_{w} \phi\right\rangle= & \int_{0}^{c+w}\left(A_{w} \phi\right)(s) d \Psi(s) \\
= & \int_{0}^{c+w}\left(-s \frac{d \phi}{d s}-x g(s) \phi(s)+x g(s) \phi(s+w)\right) \psi(s) d s \\
= & -s \phi(s) \psi(s)]_{0}^{c+w} \\
& +\int_{0}^{c+w} \phi(s)\left\{\frac{d}{d s}(s \psi(s))-x g(s) \psi(s)+x g(s-w) \psi(s-w)\right\} d s \\
= & \int_{0}^{c+w} \phi(s) d G(s),
\end{aligned}
$$

where

$$
\begin{aligned}
G(s) & =-\int_{s}^{c+w}\left(\frac{d}{d s}(s \psi(s))-x g(s) \psi(s)+x g(s-w) \psi(s-w)\right) d s \\
& =s \psi(s)-x \int_{s-w}^{s} g(\sigma) \psi(\sigma) d \sigma=s \frac{d \Psi}{d s}-x \int_{s-\psi}^{s} g(\sigma) d \Psi(\sigma) .
\end{aligned}
$$

Hence $\left\langle\Psi, A_{w} \phi\right\rangle=\langle G, \phi\rangle=\left\langle A_{w}^{*} \Psi, \phi\right\rangle$. Thus

$$
\left(A_{w}^{*} \Psi\right)(s)=G(s)=s \frac{d \Psi}{d s}-x \int_{s-w}^{s} g(\sigma) d \Psi(\sigma) .
$$

\section{References}

1. Butzer, P. L., Behrens, H.: Semigroups of operators and approximation. Berlin-Heidelberg-New York: Springer 1967

2. Cox, D. R., Miller, H. D.: The theory of stochastic processes. London: Methuen \& Co. Ltd. 1965

3. Davies, E. B.: One-parameter semigroups. London: Academic Press 1980

4. Diekmann, O., Heijmans, H. J. A. M., Thieme, H. R.: On the stability of the cell size distribution. J. Math. Biol. 19, 227-248 (1984)

5. Feller, W.; An introduction to probability theory and its applications. Vol. II (2nd ed.). New York: John Wiley \& Sons 1971

6. Fransz, H. G.: The functional response to prey density in an acarine system. PUDOC, Wageningen, 1974

7. Hale, J. K.: Theory of functional differential equations. Berlin-Heidelberg-New York: Springer 1977

8. Heijmans, H. J. A. M.: An eigenvalue problem related to cell growth. J. Math. Anal. Appl. (in press) (1985)

9. Holling, C. S.: The functional response of invertebrate predators to prey density. Mem. Ent. Soc. Canada 48, 1966 
10. Krasnosel'skii, M. A.: Positive solutions of operator equations. Groningen: Noordhoff 1964

11. Krein, M. G., Rutman, M. A.: Linear operators leaving invariant a cone in a Banach space. Amer. Math. Soc. Transl. 10, 199-325 (1962)

12. Lay, D. C.: Spectral analysis using ascent, descent, nullity and defect. Math. Ann. 184, 197-214 (1970)

13. Metz, J. A. J., van Batenburg, F. H. D.: Holling's "hungry mantid" model for the invertebrate functional response considered as a Markov process. Part 0: A survey of the main ideas and results. In: Hallam, T., S. Levin (eds.) Proc. Int. Symp. on "Mathematical Ecology", Triest, 1983

14. Metz, J. A. J., van Batenburg, F. H. D.: Holling's "hungry mantid" model for the invertebrate functional response considered as a Markov process. Part I: The full model and some of its limits. Submitted to J. Math. Biol.

15. Metz, J. A. J., van Batenburg, F. H. D.: Holling's "hungry mantid" model for the invertebrate functional response considered as a Markov process. Part II: Negligible handling time: to appear

16. Pazy, A.: Semigroups of linear operators and applications to partial differential equations. New York: Springer 1983

17. Schaefer, H. H.: Topological vector spaces. New York: Macmillan 1966

18. Taylor, A. E., Lay, D. C.: Introduction to functional analysis. New York: John Wiley \& Sons 1979

19. Webb, G. F.: Theory of nonlinear age-dependent population dynamics. to appear.

Received June 25, 1984 